\title{
The bow and sequential effects in absolute identification
}

\author{
R. DUNCAN LUCE, ROBERT M. NOSOFSKY, DAVID M. GREEN, \\ and ALBERT F. SMITH \\ Harvard University, Cambridge, Massachusetts
}

\begin{abstract}
The bow and sequential effects in absolute identification are investigated in this paper by following two strategies: (1) Experiments are performed in which sequential dependencies in signal presentations are manipulated, and (2) analyses are conducted (some of which are largely free of model-specific assumptions) which bear directly on the question of the origin of the sequential effects. The main result of the study is that absolute identification performance is greatly improved in a design in which each signal lies close to the preceding signal presented, even though the entire range of signals used is the same as in a random presentation design. This finding is consistent with the attention-band model of Luce, Green, and Weber (1976) and rejects hypotheses that suggest that the variability in the signal representation in absolute identification is a function solely of the range of signals being used. However, nonparametric analyses of sequential response errors show that a plausible assumption concerning the trialby-trial movement of the attention band provides an incomplete explanation of sequential effects in absolute identification. These results are far better explained in terms of systematic shifts of category boundaries in a Thurstonian model, as suggested by Purks, Callahan, Braida, and Durlach (1980). Experiments are also performed which suggest that memory decay is not the major factor accounting for the bow effect in absolute identification.
\end{abstract}

As one increases the number of equally spaced signals, and therefore their range, absolute identification performance deteriorates markedly except for the extreme signals (Durlach \& Braida, 1969; Weber, Green, \& Luce, 1977). This deterioration evidences itself as a deep bow in a plot of percent correct, or d', against ordinal signal position. The question is why-both why the deterioration and, given that it occurs, why performance on the extreme signals does not deteriorate as markedly. Durlach and Braida (1969) and Gravetter and Lockhead (1973) have suggested that the deterioration with range has to do with increased variability of the representations of the signals due solely to the increased range. Berliner and Durlach (1973) have suggested that the bow arises because the end signals serve as anchors and that the further a signal is from the nearest end, the more variable its representation. And Luce, Green, and Weber (1976), largely on the basis of magnitude estimation data, have suggested that both might arise from a mechanism of selective attention in the intensity dimension. Weber, Green, and Luce (1977) reported a number of studies which suggest that both the attention hypothesis and a

This work was supported in part by National Science Foundation grants to Harvard University. We would like to thank Marilyn Shaw for useful conversations and suggestions, and Diana Kornbrot for her comments on an earlier version of this article. The authors' mailing address is: Laboratory of Psychophysics, Harvard University, Cambridge, Massachusetts 02138. slightly modified version of the anchors hypothesis are consistent with the data.

There are three aspects to the attention hypothesis. First, when a signal occurs, its representation is less variable if the signal is attended than if not $^{1}$; this would arise if attention corresponded to a larger neural sample size, but that interpretation is not necessary. Second, attention tends to focus somewhat more on the end signals than elsewhere. As less variability means more accurate performance, a tendency to attend to the end signals is a way to account for the bow. And third, attention tends to focus more on the region of the last signal than elsewhere. We describe these last two hypotheses as tendencies, since neither appears to be a firm rule; indeed, as strict rules they are inconsistent. To the extent that the third hypothesis is correct, sequential effects will occur. That they do occur in absolute identification was demonstrated by Ward and Lockhead (1970), and subsequently they have been found by others.

Purks, Callahan, Braida, and Durlach (1980) have suggested, in contrast to the attention-band hypothesis, that the Thurstonian distributions that represent the signals are unaffected by the location of the previous signal, but that the category boundaries are markedly affected. They partitioned their data by the previous signal and then fit a constantvariance Thurstonian model. The separation between signal distributions was unaffected by the location of the previous signal, but the category boundaries 
were shifted away from the previous signal. So, for example, if the previous signal was at $50 \mathrm{~dB}$ and the next louder signal was at $55 \mathrm{~dB}$, then the boundary between the 50- and 55-dB signals would be located above its average value.

Although this analysis casts major doubt upon the third part of the attention explanation, it should be pointed out that the conclusions are somewhat model-dependent. Specifically, Purks et al. assume in their analysis a constant-variance Thurstonian model and allow for the sequential effects to appear only as changes in the locations of the mean scale values or in the boundary values. In contrast, the attention-band model assumes the means and boundaries to be unaffected and the variance to change locally.

To try to untangle these alternatives, two strategies are followed in this paper. One is experimentaltrying to exploit the sequential effects by manipulating the sequential dependencies in the signal presentation. The other is analytical-seeking analyses that are largely free of specific assumptions in order to examine the sequential effects in the data. Nonetheless, these analyses should bear directly on the question concerning the origin of the sequential effects. Are these effects due to reduced variance in the signal representation when the signal is closer to the preceding one, as alleged by the attentionband model, or are they due to changes in the location of the category boundaries?

\section{EXPERIMENT 1}

In the first experiment, we exploit the idea that the attention band (reduced variance in the Thurstonian representation), if it exists, tracks the previous signal. The idea is that we can constrain the sequence of signal presentations so that each signal lies close to the immediately preceding one, and yet arrange it so that, in the long run, all signals are presented equally often. More specifically, on each trial we constrain the signal to be the one just presented, the one just above it, or the one just below it, each with probability $1 / 3$, except, of course, for the two end signals (see the Method section). We refer to this as the small-step(3) condition. The sequential constraint in this condition not only causes each signal to be near the preceding one, but also makes them much more predictable a priori, reducing the possible responses from 11 alternatives in the random case to 3 in the small-step one. To disentangle these two effects, we also ran what we refer to as the largestep condition. Here, each signal was, as in the small-step case, one of three adjacent possibilities, and in the long run each signal was presented equally often. However, the three possibilities were always far from the preceding signal rather than near it. We also ran a small-step design with five adjacent signals centered on the preceding signal in order to see if performance deteriorated appreciably from the small-step(3) condition. This would be expected if the attention band was 10-20 dB wide, as has been estimated previously (Green \& Luce, 1974).

\section{Method}

The signals, wide-band noise gated on for $500 \mathrm{msec}$, were presented binaurally, in quiet, via TDH-39 headphones. The observers, one male and two females, were tested in single-wall sound-treated chambers (IAC-402A). Blocks of 100 trials were run with rests between blocks.

The stimulus set always consisted of 11 signals spaced at intervals of $5 \mathrm{~dB}$ from 40 to $90 \mathrm{~dB}$ SPL. In the random condition, successive signals were equally likely and were selected at random, independently of previous choices. In other conditions, Markov transition matrices were used to program the selection of successive signals. In these matrices, the row entry is the index of the previous stimulus, $S^{(n-1)}$, and the column entry is the index of the present stimulus, $S^{(n)}$. The large-step and small-step(3) conditions involved diagonal bands of three adjacent entries of probability $1 / 3$, with exceptions due to overflow at the end signals. The small-step(5) matrix contained a diagonal band of five adjacent entries of probability $1 / 5$. In the small-step matrices, the band was centered on the main diagonal. In the small-step(3) matrix, the end signals received entries $2 / 3,1 / 3$; in the smallstep(5) matrix, the two signals at each end received entries of $2 / 5,2 / 5,1 / 5$ and $2 / 5,1 / 5,1 / 5,1 / 5$, respectively. In the largestep matrix, the band was centered on $\left(S^{(n-1)}, S^{(n)}\right)$ pairs $(1,6)$, $(2,7), \ldots,(5,10),(6,11),(7,1),(8,2), \ldots,(11,5)$, with overflow from one end accommodated at the other extreme. For example, the entry that would have been placed in cell $(6,12)$ went in cell $(6,1)$. All these Markov chains have the property that, asymptotically, every stimulus is used equally often.

On successive days in 3-day cycles, the observers completed between 600 and 800 trials in the random, small-step(3), and large-step conditions, for a total of approximately 4,000 trials in each condition. Following this, approximately 1,500 trials were run in the small-step(5) condition. After these data were collected, it occurred to us that because the constraints of the large-step matrix might be more difficult for our observers to remember than those of the small-step(3) matrix, the large-step condition, as run, might fail to reduce response uncertainty in the way intended. Indeed, two of our observers were using a substantial number of responses which were impossible under the constraints of the matrix. Therefore, an additional 4,000 trials of large-step data were collected in which the three possible responses for each trial were printed on the terminal prior to the trial. It is these data that are presented below.

At the beginning of each block of trials, the ascending sequence of intensities was played twice. During these presentations, the identity of each signal was printed on the terminal. The observers were instructed to use the numbers from 1 (softest) through 11 (loudest) as responses to the signals, and encouraged to respond accurately.

At the beginning of each trial, the word "READY" appeared on the terminal for 500 msec. After the signal was presented, the observer entered a response; the number appeared on the screen and could be corrected prior to its being entered into the computer (PDP-15). After all observers had responded on a trial, feedback was provided and the next signal was presented following a 500-msec delay.

Observers were paid $\$ 3.50 / \mathrm{h}$ for participating in the experiment.

\section{Data Presentation}

There are several reasonable approaches to presenting the data obtained in the various conditions. 
One straightforward route is simply to compute percent correct scores or estimates of d' from the cumulative judgment matrices obtained in each condition. This approach suffers from the obvious drawback, however, that the constrained response choices in the small-step(3), small-step(5), and large-step conditions will inflate artificially subjects' performance relative to the random-step condition. For example, assume in the small-step(3) condition that stimulus 4 is presented on trial $\mathrm{n}$; if stimulus 5 is then presented on trial $\mathrm{n}+1$, and the subject judges it to be stimulus 6 , he or she will still respond 5 , since only responses 3,4 , and 5 are possible on that trial. This will increase artificially the percent correct score on stimulus 5 , or the estimate of $d_{s, 6}^{\prime}$.

In order to circumvent this difficulty, we employ an alternative method of analysis throughout this article. First, an estimate of $d_{i, i+1}^{\prime}$ is computed only for that subset of trials in which responses $i$ and $i+1$ are both available. In particular, the data are partitioned into conditional stimulus-response (S-R) matrices according to the previous signal. Values of $\mathrm{d}_{\mathrm{i}, \mathrm{i}+1}{ }^{\prime}$ are then computed from each conditional S-R matrix in which such a computation is possible, and then an overall $d_{i, i+1}^{\prime}$ value is computed by averaging each of these conditional $d_{i, i+1}^{\prime}$ estimates together. ${ }^{2}$ For example, in the small-step(3) condition, the overall $d_{4,5}^{\prime}$ is computed by estimating $d_{4,5}^{\prime}$ when the previous signal was 4 and when the previous signal was 5 , and then averaging these together. [In the smallstep(3) condition, responses $i$ and $i+1$ are both available on a given trial if and only if the previous signal was either $i$ or $i+1$.

Analogous procedures are used to compute $d_{i, i+1}^{\prime}$ in the small-step(5), large-step, and random-step conditions. The reader should note that there are 2 individual estimates of $d_{i, i+1}^{\prime}$ in the small-step(3) and large-step conditions, 4 individual estimates of $d_{i, i+1}^{\prime}$ in the small-step(5) condition, and 11 individual estimates of $d_{i, i+1}^{\prime}$ in the random-step condition which are averaged together to form each overall $d_{i, i+1}^{\prime}$ value. The more individual estimates of an overall $d_{i, i+1}^{\prime}$ value there are, the fewer data there are that go into forming each individual $d_{i, i+1}^{\prime}$ estimate.

In summary, the conditional method of analysis allows us to compare identification performance among the various conditions directly, free from contamination by the constraints on responses inherent in the sequentially constrained conditions.

\section{Results and Discussion}

Figure 1 displays the values of $\mathrm{d}_{\mathrm{i}, \mathrm{i}+1}^{\prime}$ as a function of signal i for each procedure: random, small step(3), small step(5), and large step. ${ }^{3}$ These were computed from one grand S-R matrix obtained by cumulating the data of all subjects, each subject having shown a similar pattern of results. The large-step data are from the condition in which the display indicated the three response alternatives for each trial; these data

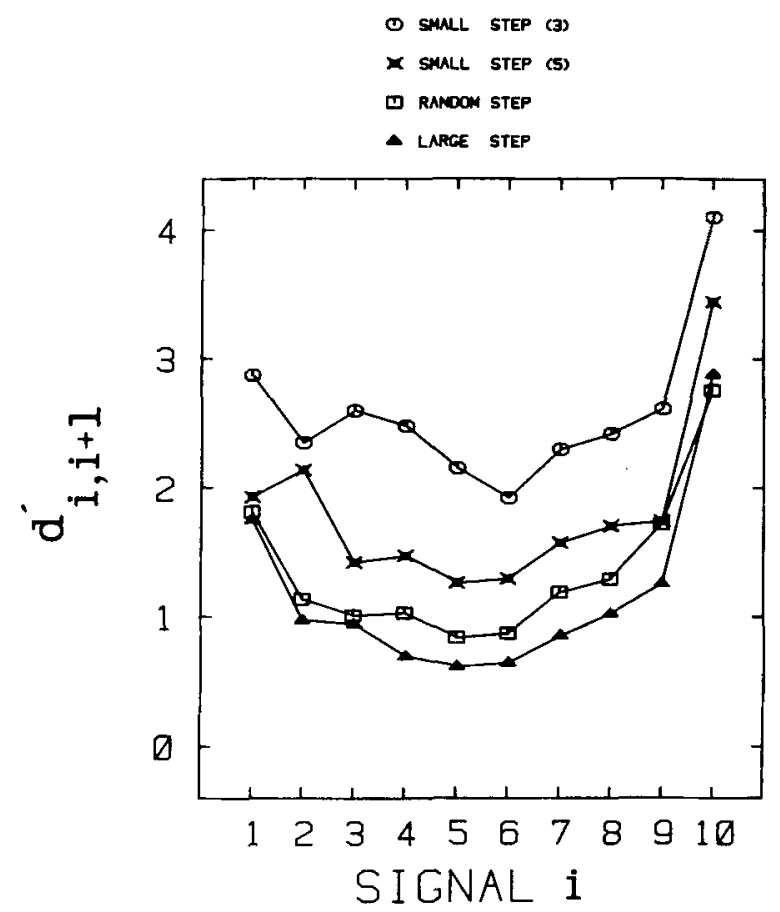

Figure 1. Values of $d_{i, i+1}^{\prime}$ as a function of signal $i$ in the random, large-step, small-step(3), and small-step(5) conditions.

are virtually indistinguishable from those collected when the alternatives were not explicitly displayed.

As is readily observed, sensitivity in the smallstep(3) condition is vastly greater than that in the random-step condition. Sensitivity in the smallstep(5) condition is substantially inferior to that in the small-step(3) condition, but is still clearly superior to the random-step performance. Sensitivity in the large-step condition is slightly, but consistently, below that obtained in the random-step condition. Each of these results is consistent with the hypothesis of an attention band, about 10-20 dB wide, which tends to track the last signal presented.

These data undercut both of the hypotheses that suggest that variability in the signal representation is a function solely of the range of signals being used. The range in each of the conditions is identical, with only the sequential dependencies in the signal presentations being manipulated, yet sensitivity in each condition is characteristically different. One might try to save the range hypothesis by arguing that the source of the effect is the range of potential signals on each trial, but this will not do because that range is equally narrow in the large-step and small-step(3) cases. Or one might try to save it by saying it is the range between successive trials that is relevant; but this becomes difficult to distinguish from the emphasis placed by the attention-band theory on the importance of sequential dependencies among signals.

Since data on absolute identification often are reported as percent correct, we show in Figure 2 our data in this form as well. Comparing Figures 1 and 2 


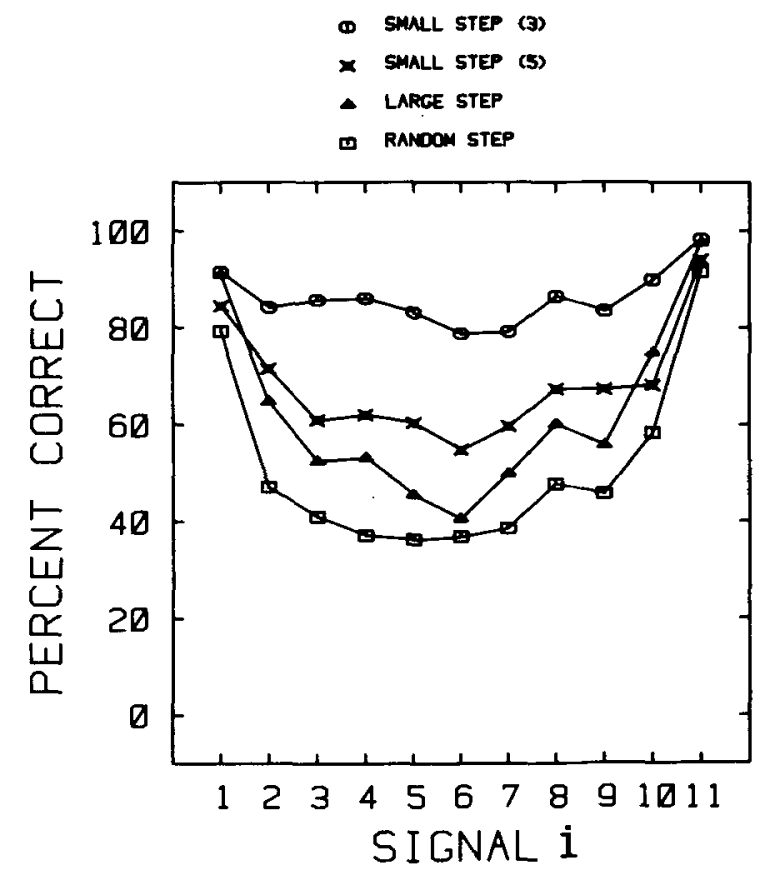

Figure 2. Percent correct scores for each signal, computed from cumulative judgment matrices in each condition.

is revealing. The first of the two most salient differences is seen in the relation between the random and large-step data: In the conditional d' analysis of Figure 1, the large-step function lies below the random one, whereas the opposite is true in the percent correct measure computed from the cumulative judgment matrix. This is clear evidence that the artificial inflation due to response restrictions of percent correct (or of $d^{\prime}$ ) computed from the cumulative matrix is not an idle concern. The second difference concerns the bowing in the data. According to the conditional $d^{\prime}$ representation, all of the conditions appear to produce the same amount of bowing, with the curves simply displaced vertically. In contrast, the percent correct representation suggests less bowing for the small-step(3) condition than for either the random or large-step ones. The apparent flattening is, at least in part, a ceiling effect of the probability measure.

The fact that, in terms of conditional $\mathrm{d}^{\prime}$, the effect of the experimental manipulations is simply to displace the bow suggests that different mechanisms may underlie the bow and the sequential effects in these absolute identification tasks. This is not a necessary conclusion, however. For example, the attention band may tend to track the last signal presented, and in addition have a special propensity to move towards the extremes of the intensity range. Another source of concern to us is that the $d_{10,11}^{\prime}$ estimate in the small-step condition is based on response probabilities that approach unity. The $d$ ' analysis assumes a very specific distribution function, namely the Gaussian. This may be a rather poor approximation in the tails of the actual distribution of the underlying decision variable. We simply do not know enough to be confident of its accuracy, and, as Wandell and Luce (1978) have demonstrated, one's conclusions can be altered radically by using other plausible distributions which differ from the Gaussian mainly in the tails. We prefer to remain cautious, therefore, regarding any final conclusions or inferences concerning the magnitude of the bow effect across the various conditions.

Discrimination strategy hypothesis. One possibility that must be considered is that the subjects' improved performance on the small-step procedure was due to their use of an alternative strategy-a strategy that is not absolute identification in the usually understood sense. As stated previously, on any given trial in the small-step(3) schedule, the stimulus was equally likely the one just presented, the one just above it, or the one just below it. Thus, instead of engaging in an absolute identification of the signals, the subjects could have simply been performing a modified discrimination task in which they judged whether the signal on trial $n+1$ was softer than, the same as, or louder than the signal on trial $n$. Their responses on trial $n+1$ would then be based on the feedback they had received on the prior trial $n$. For example, if signal 5 had been presented on trial $n$ and the subject judged the signal on trial $n+1$ to be louder, he or she would simply respond 6 . It is possible, then, that subjects' improved performance in the small-step(3) design was due solely to the fact that they were able to use a discrimination strategy.

The discrimination strategy hypothesis receives some support in Figure 3 in which we compare performance on the small-step(3) procedure with performance on the random schedule conditionalized upon prior signal. The solid line corresponds to the $\mathrm{d}_{\mathrm{i}, \mathrm{i}+1}^{\prime}$ values in the random schedule when the prior signal was either $i$ or $i+1$. As before, separate $d^{\prime}$ values were computed for each prior signal and then averaged together. (The difference between this procedure and the one used to estimate $d_{i, i+1}^{\prime}$ in Figure 1 is that we averaged over all 11 previous signals in Figure 1). The dashed line corresponds to the $d_{i, i+1}^{\prime}$ values for the small-step(3) schedule when the prior signal was (necessarily) $i$ or $i+1$. (This is the same curve as that presented in Figure 1.) As is readily observed, performance on the small-step(3) schedule is markedly superior to the random-step performance even when one restricts attention to exactly the same one-trial sequential dependencies. This result supports the hypothesis that subjects' improved sensitivity in the small-step design was due to their use of a discrimination strategy which was not used in the random-step design. 


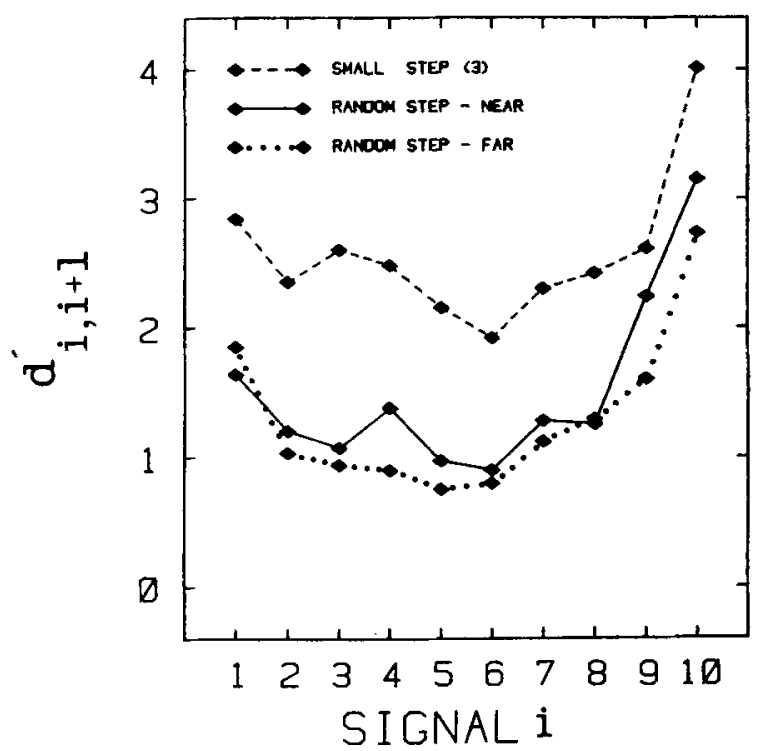

Figure 3. Values of $d_{i, j+1}^{\prime}$ for each signal pair in the small-step (3) condition, the random condition when previous signal is near, and the random condition when previous signal is far.

Intuitive and plausible as the discrimination strategy hypothesis may be, experimental evidence suggests that it is not correct. In a follow-up experiment, Nosofsky (in press) tested the hypothesis explicitly-subjects were required simply to discriminate successive signals embedded in a random-step design rather than actually to identify them. The subjects' ability to resolve adjacent signals was virtually unchanged from their performance in a random-step identification condition, and was far worse than their resolution performance in a smallstep(3) identification condition. Thus, even if subjects engaged in the hypothesized discrimination strategy in the small-step(3) condition, their superior performance in that condition must be due to something more than the opportunity to pursue that strategy. We turn, therefore, to an account of the results presented above in terms of attention bands.

Attention-band hypothesis. Suppose that attention tends to focus more in the region of the last presented signal than elsewhere, but that the shifting of the band is probabilistic, rather than strict. It is plausible that the probability that the band shifts fully to the location of the last presented signal is higher when it has a relatively short distance to move. Such a situation is guaranteed in the small-step design but not in the random-step design, in which case one should see a substantial increase in $\mathrm{d}^{\prime}$, as observed.

If, however, attention does tend to track the previous signal, one should observe at least some improvement in identification performance in the randomstep design when the previous signal is near the current $\mathrm{d}^{\prime}$ pair over performance when the previous signal is far. In Figure 3, we have plotted overall $d^{\prime}$ values for each signal pair contingent on the prior stimulus being at least two steps away from the closer signal in the pair (dotted line). For example, the overall $d_{4,5}^{\prime}$ is computed by averaging the $d_{4,5}^{\prime}$ values obtained when the prior signal was $1,2,7,8,9,10$, or 11. As can be seen in the graph, there is a small, but consistent, tendency for the $d_{i, i+1}^{\prime}$ values to be larger when the previous signal was $i$ or $i+1$ rather than when it was at least two signals away. This result is significant by a Wilcoxon test $[\mathrm{W}(10)=6.5, \mathrm{p}<.05$, nondirectional]. ${ }^{4}$ In sum, our random-step data lead us to conclude that there is some, albeit slight, improvement in identification performance measured in $d^{\prime}$ when the prior signal is near rather than far from the current signal judged. This result is in accord with the hypothesis of an attention band which tracks the prior signal in probabilistic fashion. It is in direct contrast to the result obtained by Purks et al., but draws support from the results of Siegel (1972). Nevertheless, it is a small effect and, as we now show, the shifting category boundaries found by Purks et al. are much more important as a source of sequential effects in the random-step data.

Shifting category boundaries. To review, Purks et al. found that following the presentation of a given signal, all category boundaries were shifted away from that signal. That is, all category boundaries located above the signal were shifted above their average values and all category boundaries located below the signal were shifted below their average values. We have expressed concern that the model they employ in deriving this result assumes constantvariance stimulus distributions and allows sequential effects to appear only as changes in the means of these distributions or as shifts of the category boundaries. The attention-band model, on the other hand, does not assume constant-variance stimulus distributions, and views sequential effects as resulting from local changes in variance.

We want, therefore, if at all possible, a scheme for analyzing the data that bears directly on the question quite independently of the adequacy of any model. Consider the following analysis. Let $P\left(R_{i+1}^{(n)} \mid S_{i}^{(n)}\right.$, $\left.S_{j}^{(n-1)}\right)$ be the probability of responding on trial $n$ that the signal is believed to have been $i+1, R_{i+1}^{(n)}$, when, in fact, it is $S_{i}$ and given that the signal on the preceding trial was $S_{j}$. And let $P\left(R_{i-1}^{(n)} \mid S_{i}^{(n)}, S_{j}^{(n-1)}\right)$ be the other one-step error probability under the same conditions. The attention model says that both of these probabilities of error are smaller when $i$ is close to $j$ than when $i$ is far from $j$. By previous estimates, the transition from smaller to larger should be in the neighborhood of 10-20 dB. This prediction is shown in Figure 4. In contrast, if the distributions remain the same, but the boundaries shift away from the location of the previous signal, we should have the pattern shown in Figure 5. The qualitative difference between these predictions is striking. 


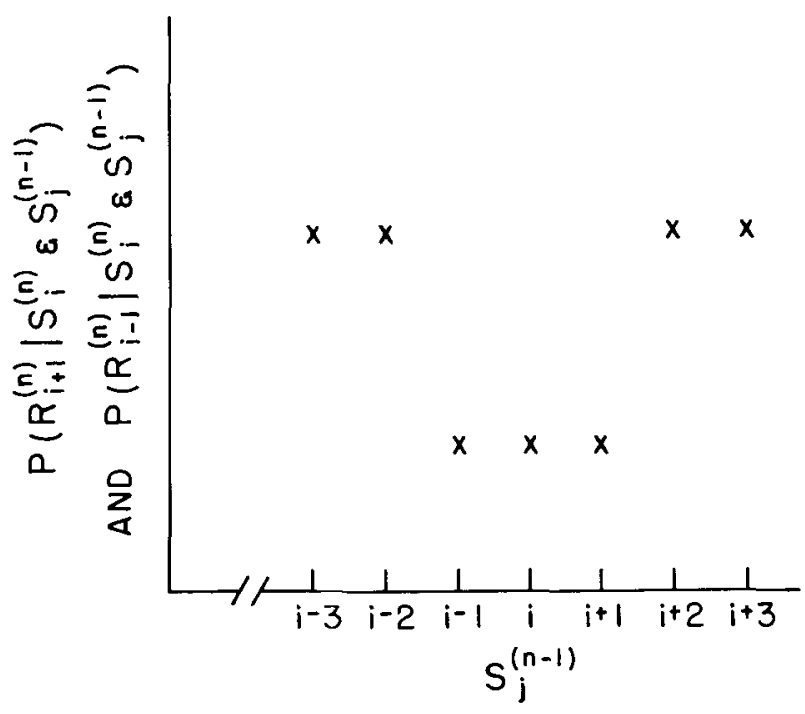

Figure 4. Schematic prediction of the sequential one-step response errors for the attention-band model. See text for a full explanation of the notation.

The data from the random-step condition, shown in Figure 6, are averaged over signals 3 to 9 and over subjects in order to have adequate sample sizes. An examination of the subjects separately and of the intermediate signals separately showed no substantial differences from the average plot. These values for end signals 1 and 2 are essentially flat at about .15 and for 10 and 11 are flat at about .10 . As is easily seen, the data are similar to Figure 5 , not to Figure 4, thereby supporting the shifting boundary idea. The hypothesis of an attention band located at the previous signal is simply inadequate to account for these sequential effects.

Table 1 presents a similar analysis for the largestep data, segregated according to whether the jump was up $\left(S^{(n-1)}<S^{(n)}\right)$ or down $\left(S^{(n-1)}>S^{(n)}\right)$. We excluded from this analysis those trials in which the current signal, $S^{(n)}$, was equal to either 1 or 11 . The left panel presents the analysis for trials in which the jump was up. Given that signal $i$ was presented on trial $n-1$, then, when the middle potential signal, $i+5$, is selected for presentation on trial $n$, the onestep error probabilities are .18 and .21 , whereas in the other two cases, the (necessarily) one-sided onestep error probabilities are twice as large. The same pattern is evident in the right panel, which presents the analysis for the trials in which the jump was down. Assuming symmetric and identical Thurstonian distributions for the three signals, this means that, in terms of shifting category boundaries, the boundaries are displaced away from the middle possible signal, and are not displaced away from the previous signal, as was found in the random case.

In Table 2, we analyzie the response errors for the small-step(3) data. When the previous signal is re- peated, the one-step error probabilities are .09 and .10 , whereas for the other two cases, the (necessarily) one-sided error rate is some $50 \%$ greater. Assuming identical and symmetric Thurstonian distributions for the three signals, one may conclude from the small-step data either that the boundaries are displaced away from the signal presented on the previous trial, as in the random condition, or that they are displaced away from the middle possibility on the current trial, as in the large-step condition. This is because, in the small-step( 3 ) condition, the middle possibility and the previous signal are always the same.

We have included in the Appendix a summary of the individual criterion shifts measured in $\mathrm{d}^{\prime}$ units for the interested reader.

Summary. In sum, the attention-band hypothesis by itself gives at best an incomplete explanation of sequential effects in absolute identification. The pat-

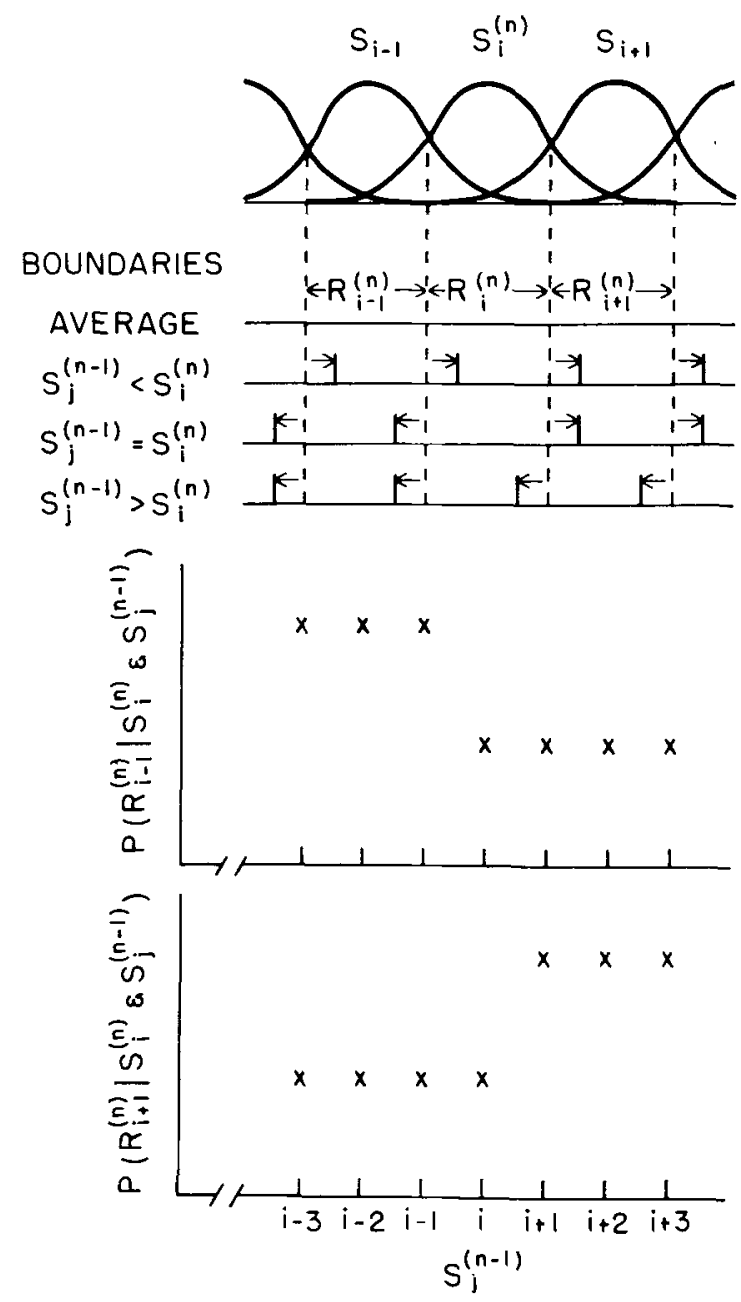

Figure 5. Top panel: Schematic illustration of the shifting category boundary finding of Purks et al. (1980). Category boundaries are pushed away from the last signal presented. Bottom panels: Predictions of the sequential one-step response errors under the rule for shifting cutegory boundaries shown above. 

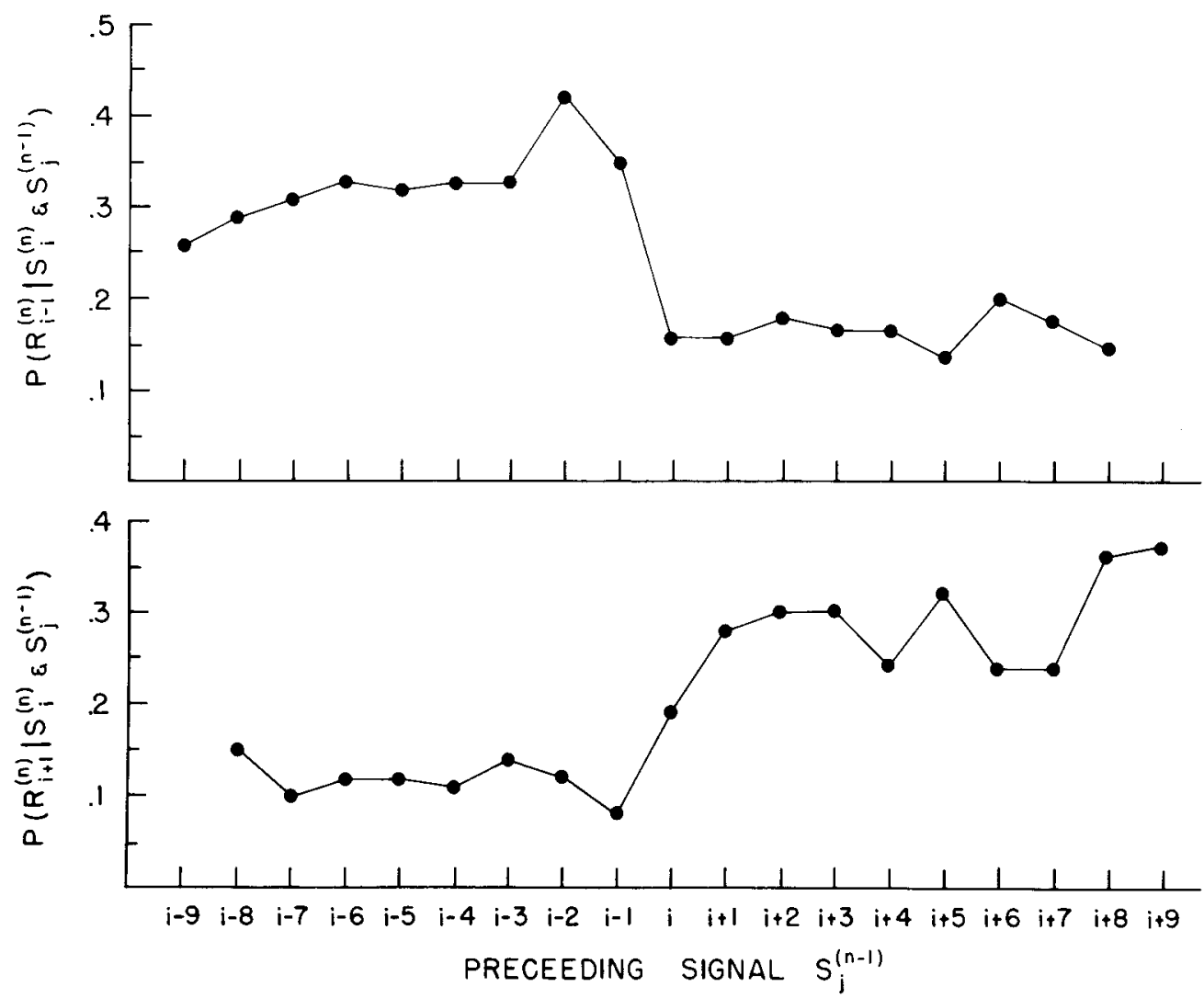

Figure 6. Results of the sequential one-step response error analysis. The data are in accord with the shifting boundary idea and are inconsistent with the prediction of the attention-band model.

tern of one-step sequential response errors observed is far better explained in terms of shifting category boundaries in a Thurstonian model, although we know of no rule that characterizes these shifts across experimental designs.

Table 1

Distribution of Responses Conditioned on Previous Signal i for Large-Step Data

\begin{tabular}{|c|c|c|c|c|c|c|c|}
\hline \multicolumn{4}{|c|}{$S^{(n-1)}<S^{(n)}$} & \multicolumn{4}{|c|}{$S^{(n-1)}>S^{(n)}$} \\
\hline \multirow[b]{2}{*}{$S^{(n)}$} & \multicolumn{3}{|c|}{ Response } & \multirow[b]{2}{*}{$S^{(n)}$} & \multicolumn{3}{|c|}{ Response } \\
\hline & $i+4$ & $\mathrm{i}+5$ & $\mathrm{i}+6$ & & $i-7$ & $i-6$ & $i-5$ \\
\hline $\mathrm{i}+4$ & .52 & .45 & .03 & $i-7$ & .57 & .39 & .04 \\
\hline $\mathrm{i}+5$ & .18 & .61 & .21 & $i-6$ & .24 & .61 & .15 \\
\hline$i+6$ & .05 & .36 & .59 & $i-5$ & .10 & .44 & .46 \\
\hline
\end{tabular}

Note-Data are averaged over previous signal $i, i=1, \ldots, 11$.

Table 2

Distribution of Responses Conditioned on Previous Signal i for Small-Step Data

\begin{tabular}{cccc}
\hline & \multicolumn{3}{c}{ Response } \\
\cline { 2 - 4 } $\mathrm{S}^{(\mathrm{n})}$ & $\mathrm{i}-1$ & $\mathrm{i}$ & $\mathrm{i}+1$ \\
\hline $\mathrm{i}-1$ & .82 & .18 & .00 \\
$\mathrm{i}$ & .09 & .81 & .10 \\
$\mathrm{i}+1$ & .00 & .14 & .86 \\
\hline
\end{tabular}

Note-Data are averaged over previous signal $i, i=3, \ldots, 9$.
The results of the various experiments do lend support, however, to the attention-band model as at least a partial explanation of absolute identification performance. Subjects' vastly improved performance in the small-step(3) design strongly supports the attention-band theory, and the results obtained in the small-step(5) and large-step conditions are also consistent with its prediction. In addition, the slightly improved performance in the random-step design when the previous signal is near rather than far from the current signal also supports a model in which attention tends to track signal presentations probabilistically.

In the remainder of the article, we examine in more detail the potential role that memory may have played in the various absolute identification experiments.

\section{EXPERIMENT 2}

A hypothesis based on memory rather than on attention or variable category boundaries was suggested to us by Marilyn Shaw. She argued that, on each trial, the subject searches his or her memory for the most recent trial on which the signal was near the current presentation. The two representations are compared and a response is selected to accord with 
the feedback given on the earlier trial. Note that it is usual in the random-step condition and inevitable in the large-step condition for the subject to have to reach several trials into the past for a relevant case. If, as everyone believes, representations in short-term memory deteriorate over time, then the subject is forced to deal with a degraded representation in these conditions. In contrast, in the small-step case it is always sufficient to work with the representation from the preceding trial.

If passage of time between presentations of signals of similar intensity is all that is important, then performance in the small-step procedure should deteriorate to the level of that observed in the large-step procedure, provided the delays between relevant signal presentations are made comparable. To check this, we used the large-step schedule but presented only those signals that fulfilled the small-step constraints, omitting all presentations of other signals. However, since the whole procedure is paced by the subjects' responses, we had to build in some time to correspond to responses to the lost signals. There are various ways this might have been done; we elected to do it by yoking together two subjects, the one run in the large-step design and the other receiving only the subset of trials fulfilling the small-step condition. We call this latter condition "yoked small step(3)."

It should be noted prior to reporting the results that, by itself, this experiment could not be definitive. If performance failed to deteriorate to the level of large steps, a memory theorist could argue that, during these pure delays, the observer engaged in some form of rehearsal. And if performance did deteriorate, an attention theorist could argue that attention might wander over long periods during which nothing was happening. A category boundary theorist could argue that the variance of the boundary locations increased with time. Nonetheless, the experiment needed to be run to see which alternative needed further investigation and amplification.

\section{Method}

The signal set and the apparatus were the same as in Experiment 1.

In each block of trials, one observer responded to 100 signals presented under the constraints of the large-step transition matrix described for Experiment 1 . The other observer received only that subset of the signals which fulfilled the constraints of the smallstep(3) transition matrix. In other words, both observers received the first trial in each block, the selection of which was unconstrained. Then the signal sequence generated according to the large-step transition matrix was presented to the one observer, and it was also monitored so that whenever a signal arose which differed by a small step from the signal last heard by the other observer, it was presented to that observer. Although most frequently only a single large-step trial intervened between successive smallstep trials, on rare occasions up to 24 large-step trials intervened. On average, the small-step observer received 27 trials to the largestep observer's 100 trials.

Two female observers, neither of whom had participated in Experiment 1, completed about 10 blocks of trials per day. For

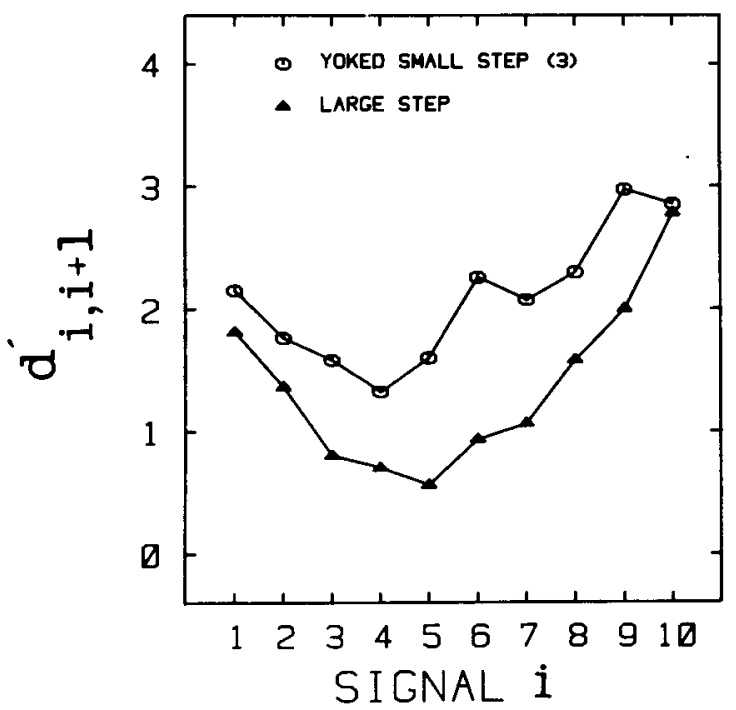

Figure 7. Values of $d_{i, i+1}^{\prime}$ as a function of signal $i$ for the largestep and yoked small-step(3) conditions.

each observer, data were collected in the large-step condition during one half of each session and in the small-step condition during the other, with the order reversed from day to day. Because we accumulated large-step data much faster than small-step data, it was necessary to run approximately 6,500 large-step trials for each observer to yield data on 1,750 yoked small-step trials.

For each observer on each of her trials, the set of responses possible for that trial under the constraints of the appropriate matrix were printed on the terminal. Otherwise, the procedure and structure of trial blocks was as described for Experiment 1.

\section{Results and Discussion}

Since the patterns of performance of the two subjects were similar, the results shown in Figure 7 are cumulated over subjects. The method of analysis was as in Experiment 1. Sensitivity in the yoked smallstep(3) condition is clearly superior to that of the same subjects in the large-step condition. We may conclude that the observed level of performance in the large-step procedure is due to something more than time since the most recent presentation of a signal of intensity similar to that of the current signal.

We also wish to note that we collected data from these same subjects in a standard small-step(3) condition and that, if anything, their performance in the yoked condition was superior to their performance in the standard condition. We conclude that mere delays between signal presentations do not contribute substantially to performance differences among conditions using our experimental paradigm.

\section{EXPERIMENT 3}

In this final experiment, we explore the possibility that much of the effect on memory has to do with intervening signals. One way to approach that would be somehow to fill the intervening time with some- 
thing to block rehearsal. An alternative, however, is to place under very close control how far into the past the observer must search for a nearby signal.

\section{Method}

Everything was as in Experiment 1, except for the nature of the sequential dependencies in the signal presentations. For each number $\mathbf{k}$, one can generate a $\mathbf{k}$-lagged small-step design as follows. The signal on trial $\mathbf{n}$ is chosen at random from among all of the signals simultaneously meeting the following conditions: It shall fulfill the small-step(3) condition for trial $n-k$, and it shall fail to satisfy that condition for all intermediate trials. For example, for lag 3 , suppose $S^{(n-3)}=2, S^{(n-2)}=9$, and $S^{(n-1)}=4$; then the possible values for $S^{(n)}$ are 1 and 2, 3 being ruled out because it meets the small-step(3) condition relative to $S^{(n-1)}$. The program was written to relax these constraints should they prove inconsistent, but that problem did not arise. On each trial, the set of three responses centered around the signal presented on trial $n-k$ appeared on the terminal screen, regardless of whether all of the corresponding signals were eligible to be presented.

One of the subjects from Experiment 2 and three new subjects participated. Approximately 1,900 responses were collected in each lag condition for two of the subjects, and approximately 2,900 responses were collected in each lag condition for the other two subjects. Insofar as was possible, lag conditions 1, 2, 3, and 4 were run on successive days in 4-day cycles.

\section{Results and Discussion}

The data for lags 1 [which is small step(3)], 2, 3, and 4 are shown in Figure 8. Again, we have combined the data of the individual subjects, since their results were similar. There seems to be little or no difference among the last three lags, which suggests that, if decreased sensitivity from the smallstep(3) condition is due to interference with memory, the effect is completely manifest when there is only one intervening signal.

A problem arises, however, in the interpretation of these data. Due to the sequential constraints embodied in the experimental procedure described above, it turns out that signals at higher lags become more and more predictable a priori. It is possible, then, that decaying memory is offset by increased signal predictability to yield equivalent performance across different lags. We therefore seek converging evidence from data we already have available from the random-step condition in Experiment 1. Specifically, we isolate those trials in the random condition which actually satisfy the sequential constraints in the k-lagged small-step design described above. For example, for lag-3 analysis, we only look at those trials for which signal $i$ is presented on trial $n$, signal $i-1$, signal $i$, or signal $i+1$ is presented on trial $n-3$, and none of these signals are presented on trial $n-1$ or trial $n-2$.

The $d_{i, i+1}^{\prime}$ values for the four lag conditions are presented in Figure 9. (Note that the lag 1 condition corresponds to the $d_{i, i+1}^{\prime}$ values already plotted in Figure 2 for the random data when the previous signal was $i$ or $i+1$.) The results, significant by a Friedman test $\left[\chi^{2}(3)=9.7, p<.05\right]$ exhibit a pattern similar to that found for the k-lagged small-step design-if anything, performance improves with in-

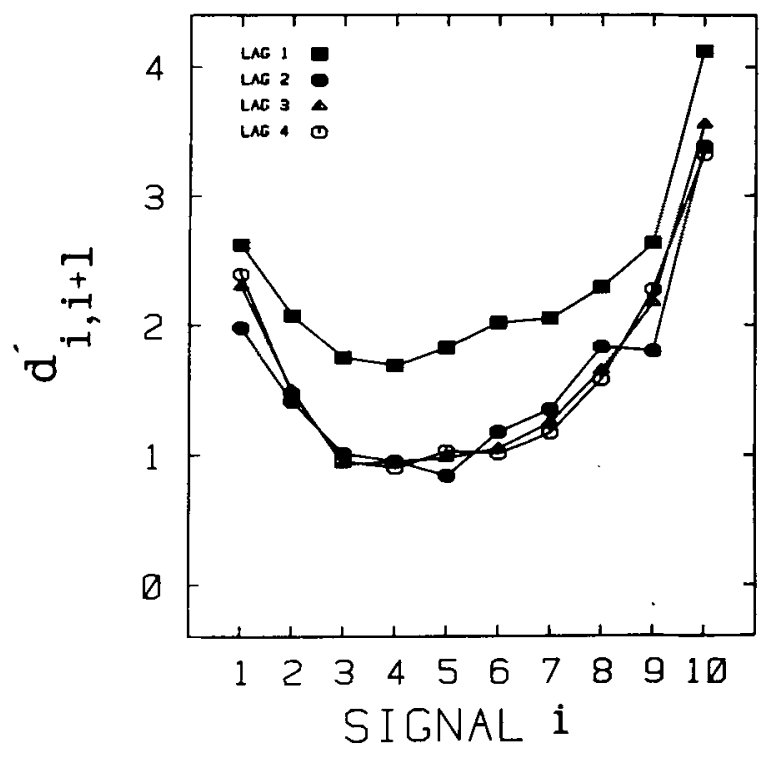

Figure 8. Values of $d_{i, i+1}^{\prime}$ as a function of signal $i$ for each lag condition (1-4).

creasing lag subsequent to lag 1 . The data from the two experiments converge on the conclusion that any memory effects are limited to one intervening trial. A similar conclusion was reached by Siegel (1972) in his study of memory effects in the absolute identification of tone frequencies.

\section{CONCLUSIONS}

Our firmest conclusion is that the account of sequential effects in absolute identification in terms of

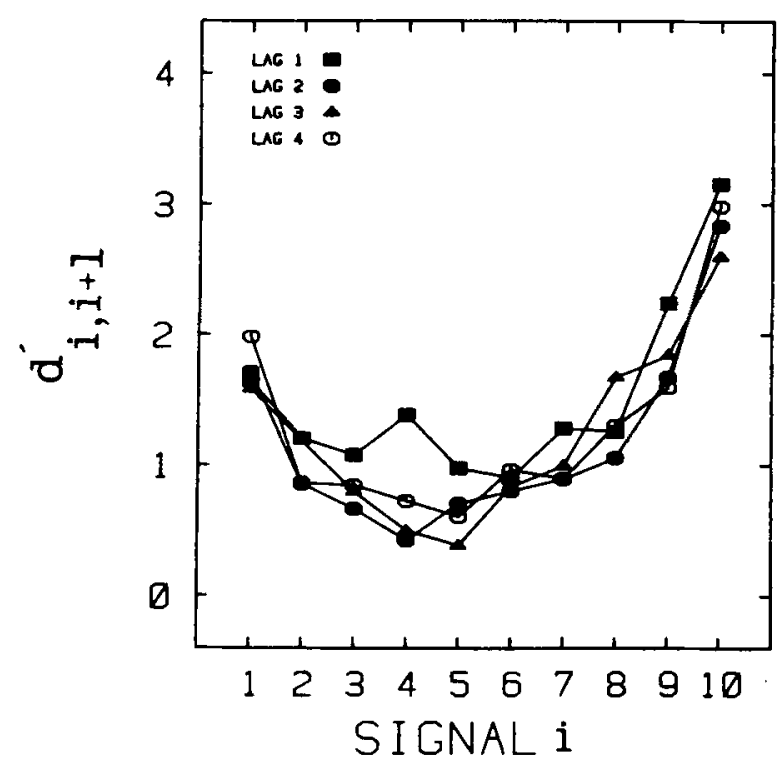

Figure 9. Values of $d_{i, t+1}^{i}$ as a function of signal $i$ for those trials in the random condition of Experiment 1 that satisfy the sequential constraints of the k-lagged small-step designs, for $k=1,2,3,4$. 
an attention band located in the region of the last presented signal is at best incomplete. Only minor (albeit significant) increases in $\mathrm{d}^{\prime}$ were observed in the random-step condition when the intensity difference between the current signal and the previous one was small as compared with when it was large. Furthermore, the pattern of sequential response errors that was observed is far better explained in terms of systematic shifts in category boundaries in a Thurstonian model. It is not clear which, if any, rule describes the shift for all cases. For example, a rule that seems to describe all but the edges of a random schedule-shift each boundary from its average position away from the previous signal-cannot account for the errors observed under the large-step, sequentially constrained presentation schedule. There the rule seems to be: Shift both boundaries away from the middle possible signal. We do not have a simple rule to account for the data from both conditions.

Despite the failure of the one-trial sequential tracking hypothesis, the attention-band model remains a viable, if incomplete, explanation of absolute identification performance. The systematic differences in the levels of sensitivity observed in the conditions of Experiment 1-small step(3), small step(5), random step, and large step-support strongly the idea of an attention band, about $10-20 \mathrm{~dB}$ wide, which tends to locate itself in that region of the intensity continuum from which signals have been recently and repeatedly presented. We need to learn a good deal more, however, about the factors that control the band's location. Among those that may be significant are the intensity difference between successive signal presentations, the degree of signal clustering, and subject expectancies.

In any case, the results observed in the sequentially constrained conditions undercut hypotheses which suggest that the variability in the signal representation is a function solely of the range of signals being used, since the range in each of these conditions was identical. The possibility that the range between successive signal presentations is the relevant factor remains as an issue for further investigation.

The possible role of memory limitations on performance in the various experimental conditions was investigated in several ways. First, the conditional d' analysis applied to the random-step data of Experiment 1 is relevant to hypotheses that emphasize the memory retention interval. That $\mathrm{d}^{\prime}$ in the randomstep condition was only slightly better than average when the previous signal was, at most, one step away from the current signal makes any hypothesis based solely on the memory retention interval difficult to defend. Second, simple time delay between signal presentations of similar intensity was shown to have, at best, minor effects in Experiment 2. Finally, in
Experiment 3, we showed that any performance deficits caused by interpolating signals of dissimilar intensity between signals of similar intensity were manifest completely in just one intervening trial:

Concerning the bow in absolute identification data, we remain unsure of its source. Limited attentional resources is still a possibility. To account for the bow, it is sufficient merely to place more of the resources on the ends of the range than in the middle, which is equivalent to saying, in the Thurstonian model, that the variances of the end signals are smaller than those of the middle ones. But that, by itself, fails to account for the fact that performance with a fixed number of equally spaced signals does not improve nearly as much with increasing range as the Thurstonian model predicts. That finding requires all variances to grow, or distances to diminish, with range, but there must be some deeper explanation for this. After all, the peripheral neural representation of a signal is almost certainly independent of the other signals being used in the experiment, and so, in particular, of the range and of whether a signal is an end one. But, if one thinks of attention as a band in intensity-like a searchlight in the representational space-that tracks signals probabilistically but with some greater tendency towards the ends of the range, then both the bow and range effects make sense. This, however, is the model Kornbrot attempted to fit to data (see Footnote 1) but rejected in favor of a singleprocess Thurstonian model.

\section{REFERENCES}

Berliner, J. E., \& Durlach, N. I. Intensity perception. IV. Resolution in roving-level discrimination. Journal of the Acoustical Society of America, 1973, 53, 1270-1287.

Durlach, N. I., \& Braida, L. D. Intensity perception. I. Preliminary theory of intensity resolution. Journal of the Acoustical Society of America, 1969, 46, 372-383.

Gravetter, F., \& Lockhead, G. R. Criterial range as a frame of reference for stimulus judgment. Psychological Review, 1973, 80, 203-216.

Green, D. M., \& LuCE, R. D. Variability of magnitude estimates: A timing theory analysis. Perception \& Psychophysics, $1974,15,291-300$.

KornBrot, D. Attention bands: Some implications for categorical judgment. British Journal of Mathematical and Statistical Psychology, 1980, 33, 1-16.

Lippmann, R. P., Braida, L. D., \& Durlach, N. I. Intensity perception V. Effect of payoff matrix on absolute identification. Journal of the Acoustical Society of America, 1976, 59, 129-134.

Luce, R. D., Green, D. M., \& Weber, D. L. Attention bands in absolute identification. Perception \& Psychophysics, 1976, 20, 49-54.

NosOFSKY, R. M. Shifts of attention in the identification and discrimination of intensity. Perception \& Psychophysics, in press.

Purks, S. R., Callahan, D. J., Braida, L. D., \& Durlach, N. I. Intensity perception. $X$. Effect of preceding stimulus on iden- 
tification performance. Journal of the Acoustical Society of America, 1980, 67, 634-637.

Siegel, W. Memory effects in the method of absolute judgment. Journal of Experimental Psychology, 1972, 94, 121-131.

WANDell, B., \& LuCE, R. D. Pooling peripheral information: Average versus extreme values. Journal of Mathematical Psychology, 1978, 17, 220-235.

WARD, L. M., \& Lockhead, G. R. Sequential effects and memory in category judgment. Journal of Experimental Psychology, $1970,84,27-34$.

Weber, D. L., GreEN, D. M., \& Luce, R. D. Effects of practice and distribution of auditory signals on absolute identification. Perception \& Psychophysics, 1977, 22, 223-231.

\section{NOTES}

1. Kornbrot (1980) has attempted to fit a particular nonconstantvariance Thurstonian version of the attention-band model to absolute identification data of Lippmann, Braida, and Durlach (1976). In particular, she assumed that the ratio of the variances on adjacent signals was a constant, as was true of the special Poisson model studied by Luce, Green, and Weber (1976). Within that special family of models, she found that the best fit to the data was with no attention bands (a single rather than a dual Thurstonian model) and with equal variances (the ratio of variances of adjacent signals is 1). This means that Kornbrot's analysis, albeit exceedingly painstaking, is limited by a rather severe prior constraint which is inherent neither in attention bands nor in nonconstant-variance models. In addition, her analysis does not take into account the existence of sequential effects, which we know to be pronounced. We hope that if the attention ideas are wrong, more direct evidence can be found.

2. Within each S-R matrix, the method used to compute each $d_{i, i+1}^{\prime}$ pair is as follows. Whenever stimulus $i+1$ is presented and a

\begin{tabular}{ccc} 
& \multicolumn{3}{c}{ Response } \\
\cline { 2 - 3 } Stimulus & $\mathrm{R} \leqslant \mathrm{i}$ & $\mathrm{R} \geqslant \mathrm{i}+1$ \\
\hline $\mathrm{i}+1$ & $\begin{array}{c}\text { Correct Rejection } \\
\text { Miss }\end{array}$ & $\begin{array}{c}\text { False Alarm } \\
\text { Hit }\end{array}$
\end{tabular}

response of $i+1$ or greater is given, it is considered a "hit," whereas a response of $i$ or less is considered a "miss." Similarly, whenever stimulus $i$ is presented and a response of $i+1$ or greater is given, it is considered a "false alarm," whereas a response of $i$ or less is a "correct rejection." Values of $d$ ' are then computed in the usual way. The procedure is summarized in the table above.

An alternative procedure (that employed by Purks et al.) would have been to compute maximum-likelihood estimates of each $\mathrm{d}^{\prime}$ pair simultaneously using a case-V (constant-variance) Thurstonian model. Although such a procedure utilizes all the information in the S-R matrix, it is not a viable approach in the present study due to the response constraints inherent in the small-step and large-step conditions. In addition, we reject the constant-variance assumption made in the model even for the random-step condition.

3. We wish to point out that, since a signal was presented only after all observers had entered a response for the previous trial, the intertrial interval in these experiments was not controlled. Indeed, it seemed to be that subjects' response times were negatively correlated with their performance across conditions. Nevertheless, we believe that the differences in intertrial intervals between conditions accounted for an extremely small proportion of the variance, and that the sequential dependencies were the major determiners of performance. Some evidence to this effect is presented in Experiment 2.

4. Using the Thurstone case- $V$ method of analysis, we find a similar pattern of results that are significant by a Wilcoxon test $[\mathrm{W}(9)=7, \mathrm{p}<.05$, directional (one tie)]

\section{APPENDIX}

In the following tables we present the criterion shifts in $\mathrm{d}^{\prime}$ units for each signal pair as a function of the previous signal presented for the random-step, small-step(3), and large-step conditions. The method for computing $\mathrm{d}_{\mathrm{i}, \mathrm{i}+1}^{\prime}$ for each signal pair is as described in Footnote 2.
Let $c_{i}$ denote the distance of the criterion from the mean of the noise distribution, and let $b_{i}$ denote the criterion shift. The values tabled below are computed by the formula

$$
\mathbf{b}_{\mathrm{i}}=\left(\mathrm{c}_{\mathrm{i}}-\mathrm{d}_{\mathrm{i}, \mathrm{i}+1}^{\prime} / 2\right) / \mathrm{d}_{\mathrm{i}, \mathrm{i}+\mathbf{1}}^{\prime} \text {. }
$$

Table A1

Random Step Criterion Shifts $\left(b_{\mathbf{i}}\right)$

\begin{tabular}{|c|c|c|c|c|c|c|c|c|c|c|}
\hline \multirow{2}{*}{$\begin{array}{c}\text { Previous } \\
\text { Signal }\end{array}$} & \multicolumn{10}{|c|}{ i } \\
\hline & 1 & 2 & 3 & 4 & 5 & 6 & 7 & 8 & 9 & 10 \\
\hline 1 & .16 & .56 & .35 & .53 & .16 & .69 & .50 & .52 & .19 & -.00 \\
\hline 2 & -.21 & .20 & .70 & .44 & .75 & .51 & .41 & .33 & .19 & -.08 \\
\hline 3 & -.17 & -.27 & .26 & .44 & .51 & .38 & .34 & .45 & .19 & .00 \\
\hline 4 & -.04 & -.27 & -.25 & -.03 & .46 & .47 & .47 & .44 & .30 & -.01 \\
\hline 5 & -.03 & -.21 & -.03 & -.16 & .37 & .49 & .41 & .36 & .30 & -.02 \\
\hline 6 & .03 & -.10 & -.22 & -.52 & -.20 & .53 & .39 & .37 & .29 & .02 \\
\hline 7 & .02 & -.36 & -.28 & -.46 & -.41 & -.18 & .24 & .37 & .36 & .01 \\
\hline 8 & -.05 & -.22 & -.45 & -.20 & -.40 & -.18 & -.10 & .44 & .44 & .10 \\
\hline 9 & -.01 & -.15 & -.25 & -.44 & -.18 & -.35 & -.17 & .06 & .29 & .18 \\
\hline 10 & -.22 & -.50 & -.28 & -.36 & -.41 & -.41 & -.20 & .08 & -.05 & -.15 \\
\hline 11 & .04 & -.54 & -.50 & -.04 & -.31 & -.03 & .07 & .21 & -.09 & -.08 \\
\hline
\end{tabular}


Table A2

Large-Step Criterion Shifts $\left(\mathbf{b}_{\mathbf{j}}\right)$

\begin{tabular}{|c|c|c|c|c|c|c|c|c|c|c|c|c|}
\hline \multirow{2}{*}{$\begin{array}{c}\text { Previous } \\
\text { Signal }\end{array}$} & \multicolumn{5}{|c|}{$\mathbf{i}$} & \multirow{2}{*}{$\begin{array}{l}\text { Previous } \\
\text { Signal }\end{array}$} & \multicolumn{6}{|c|}{$\mathrm{i}$} \\
\hline & 1 & 2 & 3 & 4 & 5 & & 5 & 6 & 7 & 8 & 9 & 10 \\
\hline $\begin{array}{l}i+6 \\
i+7\end{array}$ & $\begin{array}{l}.30 \\
.01\end{array}$ & $\begin{array}{r}.69 \\
-.18\end{array}$ & $\begin{array}{r}.64 \\
-.37\end{array}$ & $\begin{array}{r}.73 \\
-.24\end{array}$ & .87 & $\begin{array}{l}i-5 \\
i-4\end{array}$ & -.54 & $\begin{array}{r}.59 \\
-1.24\end{array}$ & $\begin{array}{r}.13 \\
-.85\end{array}$ & $\begin{array}{r}.39 \\
-.48\end{array}$ & $\begin{array}{r}.12 \\
-.42\end{array}$ & $\begin{array}{l}-.11 \\
-.12\end{array}$ \\
\hline
\end{tabular}

Table A3

Small-Step Criterion Shifts $\left(\mathbf{b}_{\mathbf{j}}\right)$

\begin{tabular}{|c|c|c|c|c|c|c|c|c|c|c|}
\hline \multirow{2}{*}{$\begin{array}{c}\text { Previous } \\
\text { Signal }\end{array}$} & \multicolumn{10}{|c|}{$\mathbf{i}$} \\
\hline & 1 & 2 & 3 & 4 & 5 & 6 & 7 & 8 & 9 & 10 \\
\hline $\begin{array}{l}i \\
i+1\end{array}$ & $\begin{array}{r}.01 \\
-.08\end{array}$ & $\begin{array}{l}-.05 \\
-.12\end{array}$ & $\begin{array}{l}-.02 \\
-.10\end{array}$ & $\begin{array}{l}-.02 \\
-.07\end{array}$ & $\begin{array}{r}.09 \\
-.16\end{array}$ & $\begin{array}{r}.07 \\
-.12\end{array}$ & $\begin{array}{l}-.01 \\
-.08\end{array}$ & $\begin{array}{r}.15 \\
-.01\end{array}$ & $\begin{array}{r}.08 \\
-.02\end{array}$ & $\begin{array}{l}-.01 \\
-.00\end{array}$ \\
\hline
\end{tabular}

(Manuscript received March 15, 1982;

revision accepted for publication September 13, 1982.) 